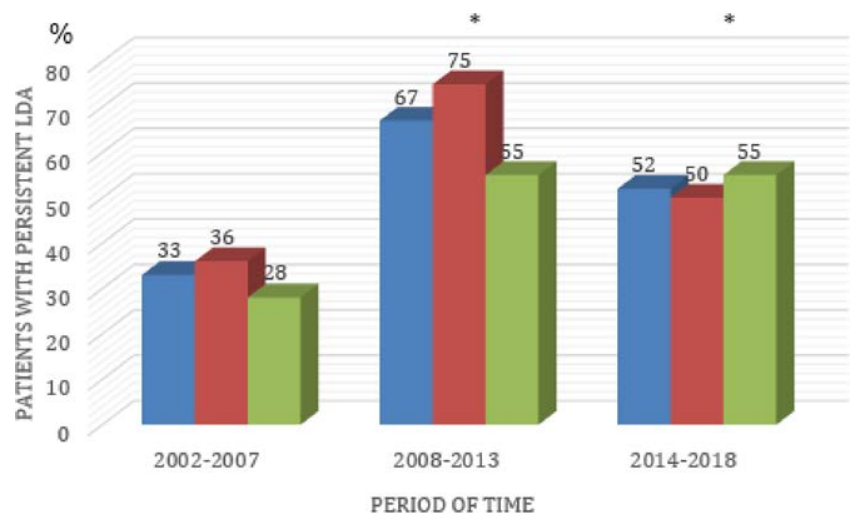

- Total mpPA maxPsA

Figure 1. Patients achieving persistent-LDA during the $1^{\text {st }}$ year of biological therapy, stratified by period of time and by disease. * Statistically significant difference with respect to $p 1$.

Disclosure of Interests: Diego Benavent: None declared, Victoria Navarro-Compán Consultant of: Abbvie, Lilly, Novartis, Pfizer, UCB, Speakers bureau: AbbVie, MSD, Lilly, Novartis, Pfizer, UCB, Irene Monjo: None declared, Marta Novella-Navarro: None declared, Alejandro Balsa Grant/research support from: BMS, Roche, Consultant of: AbbVie, Gilead, Lilly, Pfizer, UCB, Sanofi, Sandoz, Speakers bureau: AbbVie, Lilly, Sanofi, Novartis, Pfizer, UCB, Roche, Nordic, Sandoz, Chamaida Plasencia: None declared DOI: 10.1136/annrheumdis-2020-eular.1710

\section{AB0742 CONVENTIONAL SYNTHETIC DMARDS IN PSORIATIC ARTHRITIS - CHANGING PRACTICE IN BIOLOGIC ERA: REAL-LIFE RESULTS FROM HURBIO-PSA REGISTRY}

E. Bilgin ${ }^{1}$, E. Duran ${ }^{1}$, E. C. Bolek ${ }^{1}$, B. Farisoğulları ${ }^{1}$, G. K. Yardımcı ${ }^{1}$, L. Kılıç ${ }^{1}$, A. Akdoğan ${ }^{1}$, s.. A. Bilgen ${ }^{1}$, O. Karadag ${ }^{1}$, A. I. Ertenli', S. Kiraz ${ }^{1}$, U. Kalyoncu' ${ }^{1}$ Hacettepe University, Rheumatology, Ankara, Turkey

Background: Conventional synthetic disease modifiying anti-rheumatic drugs (csDMARDs) are recommended as the first-line treatment options for most of the psoriatic arthritis (PsA) patients. In the last two decades, biologic drugs become more accessible and their percentage in the daily practice is increasing continuously. However, how they influenced the utilization of csDMARDs still remains unknown, yet.

Objectives: To determine the utilization rates of PsA patients before, after and at the starting of biologic DMARDs

Methods: We analyzed all patients who received at least 1 dose of biologic DMARDs, registered to HURBIO-PsA database, and who have complete data regarding csDMARD use before (ever), after (at last control visit) and the starting of biologic DMARD. Methotrexate, leflunomide and sulphasalasine were the csDMARDs recorded. Demographic data of these patients were also recorded. Results: A total of 426 (70\% female) PsA patients was included. Mean age and mean PsA disease duration were $48 \pm 12.4$ and $9.3 \pm 8.3$ years, respectively. Mean duration of CSDMARD utilization before bDMARDs was $5.8 \pm 5.1$ years, and mean follow-up duration under bDMARDs was $3.7 \pm 2.5$ years. Distribution of the bDMARDs that ever-prescribed as follows: adalimumab $273(64.2 \%)$, etanercept $125(29.3 \%)$, certolizumab pegol $103(24.2 \%)$, infliximab $102(24.0 \%)$, secukinumab $63(14.8 \%)$, golimumab 55 (12.9\%), ustekinumab 24 (5.6\%) and tofacitinib 11 (3.4\%). Percentage of each csDMARDs used before (ever used), after (at last control visit) and at the starting of biologic DMARDs were given in Figure. Also the percentage of patients using csDMARD as monotherapy and combination therapy were given in Figure.

Conclusion: csDMARDs particularly sulphasalazine and methotrexate were important treatment options before bDMARD period, however they (particularly SSZ) were usually discontinued after bDMARD initiation. Rate of concomitant csDMARDs use remains relatively stable after starting the bDMARDs. Besides, rate of concomitant mono/csDMARD use is significantly higher after bDMARD initiation, in contrast to pre-bDMARD period.

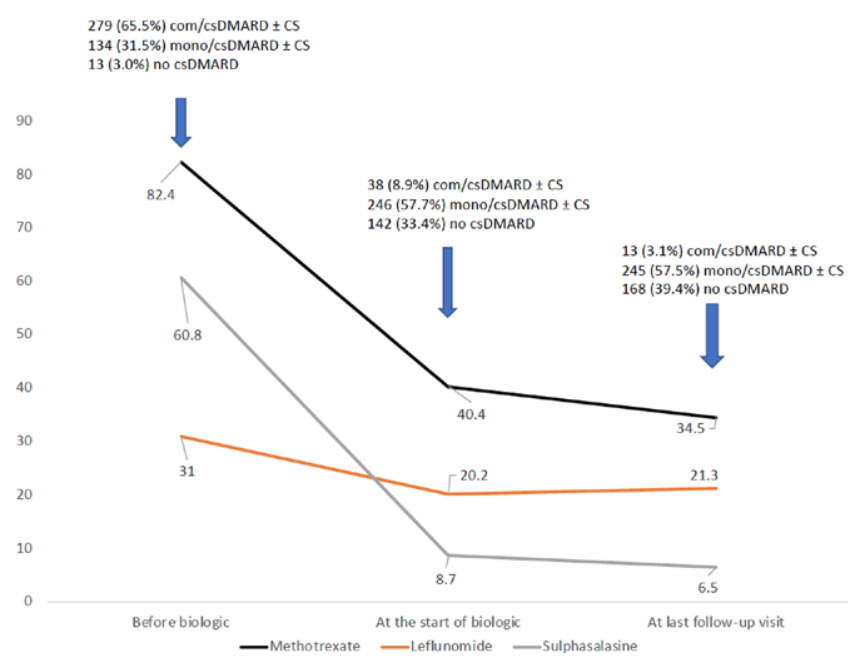

Figure. Percentage of each csDMARDs, and mono or combination of csDMARDs used before (ever used), after (at last control visit) and the starting of biologic DMARDs (com/ CSDMARD: combination CSDMARD; mono/cSDMARD: monotherapy of CSDMARD; $C S D$ MARD: conventional synthetic disease modifying antirheumatic drug).

Disclosure of Interests: Emre Bilgin: None declared, Emine Duran: None declared, Ertugrul Cagri Bolek: None declared, Bayram Farisoğulları: None declared, Gözde Kübra Yardımcı: None declared, Levent Kılıç: None declared, Ali Akdoğan: None declared, Şule Apraş Bilgen: None declared, Omer Karadag: None declared, Ali ìnsan Ertenli: None declared, Sedat Kiraz: None declared Umut Kalyoncu Consultant of: Abbvie, Amgen, Janssen, Lilly, Novartis, UCB DOI: 10.1136/annrheumdis-2020-eular.4735

\section{AB0743 DISEASE CHARACTERISTICS OF PSORIATIC ARTHRITIS PATIENTS MAY DIFFER ACCORDING TO AGE AT PSORIASIS ONSET: CROSS- SECTIONAL ANALYSIS OF PSORIATIC ARTHRITIS- INTERNATIONAL DATABASE}

E. Bilgin ${ }^{1}$, Ö. Bayındır², E. Kasapoğlü ${ }^{3}$, S. Bakırcı ${ }^{4}$, D. Solmaz², G. Kimyon ${ }^{5}$, A. Doğru ${ }^{6}$, E. Dalkılıç ${ }^{7}$ C. Özişler ${ }^{1}$, M. Can $^{3}$, S. Akar ${ }^{2}$, E. F. Tarhan ${ }^{8}$, Ş. Yavuz ${ }^{3}$, L. Kılıç ${ }^{1}$, O. Küçükşahin ${ }^{1}$, A. Omma ${ }^{1}$, E. Gönüllü ${ }^{9}$, F. Yıldız $^{10}$, D. Ersözlü ${ }^{11}$, A. Tufan ${ }^{1}$, M. Çınar ${ }^{1}$, A. Erden ${ }^{1}$, S. Yılmaz ${ }^{12}$, S. Pehlevan ${ }^{3}$, T. Duruöz ${ }^{3}$, S. Aydin ${ }^{13}, U$. Kalyoncu ${ }^{1}$ on behalf of Psoriatic Arthritis-International Database. ${ }^{1}$ PSART-ID, Ankara, Ankara, Turkey; ${ }^{2}$ PSART-ID, Izmir, Izmir, Turkey; ${ }^{3} P S A R T-I D$, istanbul, istanbul, Turkey; ${ }^{4}$ PSART-ID, Antalya, Antalya, Turkey; ${ }^{5}$ PSART-ID, Hatay, Hatay, Turkey; ${ }^{6} P S A R T-I D$, Isparta, Isparta, Turkey; ${ }^{7} P S A R T-I D$, Bursa, Bursa, Turkey; ${ }^{8} P S A R T-I D$, Muğla, Muğla, Turkey; ${ }^{9} P S A R T-I D$, Sakarya, Sakarya, Turkey; ${ }^{10}$ PSART-ID, Kahramanmaraş, Kahramanmaraş, Turkey; ${ }^{11}$ PSART-ID, Adana, Adana, Turkey; ${ }^{12}$ PSART-ID, Konya, Konya, Turkey; ${ }^{13}$ PSART-ID, Ottawa, Ottawa, Canada

Background: Psoriasis and psoriatic arthritis (PsA) are heterogenous diseases with various disease manifestations and phenotypes. Psoriasis has a bimodal age of onset being early (before the age of 40, type 1) and late. The impact of this classification on the PsA features is not well understood.

Objectives: To compare the PsA characteristics of patients with early- and late-onset psoriasis in a large, multicenter database

Methods: PSART-ID (Psoriatic Arthritis-International Database) is a prospective, multicenter web-based registry (www.trials-network.org) of patients with PsA A detailed data collection was performed including demographics (sex, age, duration of education, smoking status, BMI), skin features (psoriasis onset date, type, initially involved site of skin, nail involvement (ever) and family history) and PsA characteristics (type of articular involvement and presence of axial, dactylitis (ever), enthesitis (ever), family history) and indices for disease activity and function (DAPSA, Leeds enthesitis index, BASDAI, BASFI, patient and physician global assessment, pain, HAQ-DI). We grouped according to the age at psoriasis onset (early onset, psoriasis before the age of 40 (EOPsO); late-onset, psoriasis after the age of 40 (LOPsO)), patient and disease characteristics of the groups were compared (1). Due to the differences among groups, following adjustments weer made: $\mathrm{BMI}$ for age, nail involvement for $\mathrm{PsO}$ disease duration, axial PsA for PsA disease duration.

Results: A total of 1634 (62.8\% females; EOPsO, 1108 (67.8\%); LOPsO, 526 $(32.2 \%))$ patients with PsA was recruited. Rate of over-weight patients was higher in LOPsO group $(66.8 \%$ vs. $86.8 \%, \mathrm{p}<0.001$; adjusted for age - aOR $1.55(1.11-2.20 ; \% 95 \mathrm{Cl}))$. The EOPsO group had the scalp involvement as the initial site of skin disease more often than the LOPsO group $(56.7 \%$ 
vs. $43.0 \%, p<0.001$ ), whereas extremity involvement was more frequent as the initial finding in the LOPsO group (EOPsO vs. LOPsO $63.8 \%$ vs. $74.2 \%$, $\mathrm{p}<0.001$ ). Nail involvement (ever) was more prominent in EOPsO group, however, the significance was disappeared when adjusted for psoriasis duration. Interaction between gender and both axial disease and psoriatic disease family history were found (axial disease in man; EOPsO vs LOPsO; $38.0 \%$ vs. $25.4 \%$; $p=0.005$; adjusted for PsA duration - aOR 0.56 (0.38-0.84; $\% 95 \mathrm{Cl}) / /$ psoriatic disease family history in females; EOPsO vs LOPsO; $39.5 \%$ vs. $30.1 \%$; $p=0.003$; OR $0.65(0.50-0.86$; $\% 95 \mathrm{Cl}))$. Duration between $\mathrm{PsO}$ and PsA was significantly longer in EOPsO group (148 vs. 24 months, $\mathrm{p}<0.001)$. In EOPsO group, more patients had PsO preceeding PsA than LOPsO group ( $81.8 \%$ vs. $60.6 \%, p<0.001)$, however, synchronous disease -defined as the diagnosis of PsO and PsA within the same year- was more common in LOPsO group ( $16.6 \%$ vs. $30.3 \%, p<0.001$ ) (Table 1). Psoriatic disease activity parameters, patient and physician reported outcomes and HAQ-DI scores were similar in both groups.

Conclusion: Clinical features of PsA may be affected by the age at the onset of psoriasis. As the genetic background is different in early and late-onset psoriasis, this may suggest a different pathogenetic mechanism based on the psoriasis phenotype, also affecting the PsA features. Further prospective studies are needed to define whether the classification of PsA requires including psoriasis phenotypes as well.

References:

[1] Henseler T, Christophers E. Psoriasis of early and late onset: characterization of two types of psoriasis vulgaris. J Am Acad Dermatol. 1985;13(3):450-6.

Disclosure of Interests: Emre Bilgin: None declared, Özün Bayındır: None declared, esen kasapoğlu: None declared, Sibel Bakırcı: None declared, Dilek Solmaz: None declared, Gezmiş Kimyon: None declared, Atalay Doğru: None declared, Ediz Dalkılıç: None declared, Cem Özişler: None declared, Meryem Can: None declared, Servet Akar: None declared, Emine Figen Tarhan: None declared, Şule Yavuz: None declared, Levent Kılıç: None declared, Orhan Küçükşahin: None declared, Ahmet Omma: None declared, Emel Gönüllü: None declared, Fatih Yıldız: None declared, Duygu Ersözlü: None declared, abdurrahman tufan: None declared, Muhammet Çınar: None declared, Abdulsamet Erden: None declared, Sema Yılmaz: None declared, Seval Pehlevan: None declared, Tuncay Duruöz: None declared, Sibel Aydin: None declared, Umut Kalyoncu Consultant of: Abbvie, Amgen, Janssen, Lilly, Novartis, UCB DOI: 10.1136/annrheumdis-2020-eular.519

Table 1. Comparison of psoriatic arthritis patients' characteristics according to age at psoriasis onset

\begin{tabular}{|c|c|c|c|c|c|}
\hline Variable & $\begin{array}{c}\text { EOPsO } \\
(\mathrm{n}=1108, \\
67.8 \%)\end{array}$ & $\begin{array}{l}\text { LOPsO } \\
(\mathrm{n}=526, \\
32.2 \%) \\
\end{array}$ & $\begin{array}{c}\text { Overall } \\
(\mathrm{n}=1634)\end{array}$ & $\begin{array}{l}\text { Odds ratio }(\% 95 \\
\text { CI) }\end{array}$ & P value \\
\hline Age, mean (SD) & $41.5(11.8)$ & $58.3(8.3)$ & $46.9(13.3)$ & - & $<0.001$ \\
\hline Female, n (\%) & $674(60.8)$ & $352(66.9)$ & $1026(62.8)$ & $0.76(0.61-0.95)$ & 0.017 \\
\hline BMI, mean (SD) & $27.6(5.2)$ & $29.5(5.0)$ & $28.2(5.2)$ & & $<0.001$ \\
\hline$\geq 25, \mathrm{n}(\%)$ & $736(66.8)^{\prime}$ & $454(86.8)$ & $1190(73.3)$ & $\begin{array}{l}3.26(2.46-4.32) \\
1.55(1.11-2.20\end{array}$ & $\begin{array}{r}<0.001 \\
0.011\end{array}$ \\
\hline \multicolumn{6}{|c|}{ Psoriasis (PsO) } \\
\hline Age at diagnosis, mean (SD) & $23.5(9.4)$ & $50.4(7.2)$ & $32.1(15.3)$ & - & $<0.001$ \\
\hline $\begin{array}{l}\text { Disease duration, mean (SD) } \\
\text { (year) }\end{array}$ & $18.1(12.2)$ & $7.8(6.5)$ & $14.7(11.7)$ & - & $<0.001$ \\
\hline Initial scalp inv., n(\%)* & $560(56.7)$ & $193(43.0)$ & $753(52.5)$ & $1.74(1.32-2.18)$ & $<0.001$ \\
\hline Initial torso inv. $\mathbf{n}(\%)^{*}$ & $208(21.1)$ & $84(18.7)$ & $292(20.3)$ & $1.16(0.87-1.54)$ & 0.32 \\
\hline Initial extremity inv. $\mathbf{n}(\%)^{*}$ & $629(63.8)$ & $333(74.2)$ & $962(67)$ & $0.61(0.47-0.78)$ & $<0.001$ \\
\hline Initial genital inv. $\mathbf{n}(\%)^{*}$ & $73(7.4)$ & $29(6.5)$ & $102(7.1)$ & $1.15(0.74-1.80)$ & 0.52 \\
\hline Plaque psoriasis $(+), \mathrm{n}(\%)^{* *}$ & $649(80.8)$ & $295(80.2)$ & $944(80.6)$ & $0.95(0.70-1.30)$ & 0.81 \\
\hline $\begin{array}{l}\text { Pustuler psoriasis }(+) \\
\text { n(\%) }\end{array}$ & $144(17.9)$ & $69(18.8)$ & $213(18.2)$ & $1.05(0.76-1.45)$ & 0.73 \\
\hline $\begin{array}{l}\text { Nail involvement (ever), } \\
\text { n(\%) }\end{array}$ & $552(50.0)$ & $222(42.2)$ & $774(47.5)$ & $\begin{array}{l}1.36(1.10-1.68) \\
0.99(0.78-1.25)^{\prime \prime}\end{array}$ & $\begin{array}{c}0.003 \\
0.96\end{array}$ \\
\hline \multicolumn{6}{|c|}{ Psoriatic Arthritis (PsA) } \\
\hline Age at diagnosis, mean (SD) & $35.8(11.2)$ & $54.2(8.4)$ & $41.7(13.4)$ & - & $<0.001$ \\
\hline Disease duration, mean (SD) & $5.7(7.6)$ & $4.2(5.2)$ & $5.2(7.0)$ & - & $<0.001$ \\
\hline Monoarthritis, $\mathbf{n}(\%)$ & $30(2.7)$ & $20(3.8)$ & $50(3.1)$ & $1.42(0.80-2.53)$ & 0.22 \\
\hline Oligoarthritis, n(\%) & $344(31.1)$ & $163(31.2)$ & $507(31.1)$ & $1.01(0.82-1.26)$ & 0.98 \\
\hline Polyarthritis, n(\%) & $542(49.1)$ & $246(47.0)$ & $789(48.5)$ & $0.90(0.72-1.11)$ & 0.42 \\
\hline Arthritis mutilans, n(\%) & $3(0.3)$ & $1(0.2)$ & $4(0.2)$ & $0.70(0.07-6.78)$ & 0.76 \\
\hline \multicolumn{6}{|l|}{ Axial disease, $\mathbf{n}(\%)$} \\
\hline - Female & $187(27.8)$ & $78(22.3)$ & $265(25.9)$ & $0.76(0.56-1.03)^{:}$ & 0.08 \\
\hline - Male & $164(38.0)$ & $44(25.4)$ & $208(34.4)$ & $0.56(0.38-0.84)^{:}$ & 0.005 \\
\hline DIP involvement, n(\%) & $164(14.8)$ & $76(14.5)$ & $240(14.7)$ & $0.97(0.72-1.30)$ & 0.86 \\
\hline Dactylitis (ever), n(\%) & $245(23.4)$ & $123(24.7)$ & $368(22.9)$ & $1.07(0.83-1.38)$ & 0.56 \\
\hline Enthesitis (ever), n(\%) & $255(25.0)$ & $129(26.3)$ & $384(23.5)$ & $1.07(0.84-1.37)$ & 0.56 \\
\hline CASPAR, mean (SD) & $3.64(1.1)$ & $3.58(1.1)$ & $3.62(1.1)$ & -. & 0.25 \\
\hline CASPAR (+), n(\%) & $959(86.7)$ & $444(84.7)$ & $1403(85.9)$ & $0.85(0.63-1.14)$ & 0.28 \\
\hline \multicolumn{6}{|c|}{ Psoriatic disease } \\
\hline PsO before PsA, n(\%) & & & & & $<0.001$ \\
\hline - Yes & & & & $3.14(2.48-3.98)$ & $<0.001$ \\
\hline - No & $20(1.8)$ & $51(9.6)$ & $71(4.3)$ & $0.17(0.10-0.29)$ & $<0.001$ \\
\hline - Synchronous & $161(14.6)$ & $149(28.3)$ & $310(19.1)$ & $0.43(0.34-0.55)$ & $<0.001$ \\
\hline \multicolumn{6}{|l|}{ Family history, n(\%) } \\
\hline - Female & $266(39.5)$ & $106(30.1)$ & $372(36.3)$ & $0.65(0.50-0.86)$ & 0.003 \\
\hline - Male & $127(29.3)$ & $52(29.9)$ & $179(29.4)$ & $1.03(0.70-1.51)$ & 0.87 \\
\hline $\begin{array}{l}\text { Duration between PsO and } \\
\text { PsA, (month), med (min- } \\
\text { max) }\end{array}$ & $\begin{array}{l}148(-218, \\
797)\end{array}$ & $\begin{array}{c}24(-240 \\
313)\end{array}$ & $\begin{array}{c}80(- \\
240,797)\end{array}$ & - & $<0.001$ \\
\hline
\end{tabular}

BMI: Body mass index, CASPAR: CIASsification criteria for Psoriatic Arthritis, DIP: Distal Interphalangeal

\section{$\mathrm{AB} 0744$ PSORIATIC DISEASE AND BODY COMPOSITION: A SYSTEMATIC REVIEW OF THE LITERATURE}

T. Blake ${ }^{1,2}$, N. Gullick ${ }^{1}{ }^{1}$ University Hospitals Coventry and Warwickshire NHS Trust, Rheumatology, Coventry, United Kingdom; ${ }^{2}$ University of Warwick, Coventry, United Kingdom

Background: There is emerging evidence of a relationship between psoriasis and metabolic syndrome, increased prevalence of cardiovascular risk factors and adverse cardiovascular outcomes. Obesity is a risk factor for psoriatic disease itself but there has been less emphasis on body mass alterations and the distribution between separate compartments: fat-free tissue (lean body mass), extracellular water and adipose tissue. Body composition is a term used to describe the percentages of fat, bone, water and muscle in human bodies and is an emerging tool in assessment of physical fitness and metabolic health, although its role in disease is less well appreciated.

Objectives: To identify whether psoriatic patients, including those with psoriatic arthritis, confer specific and recognizable body composition changes compared

Figure 1. Flow diagram of articles included in this review

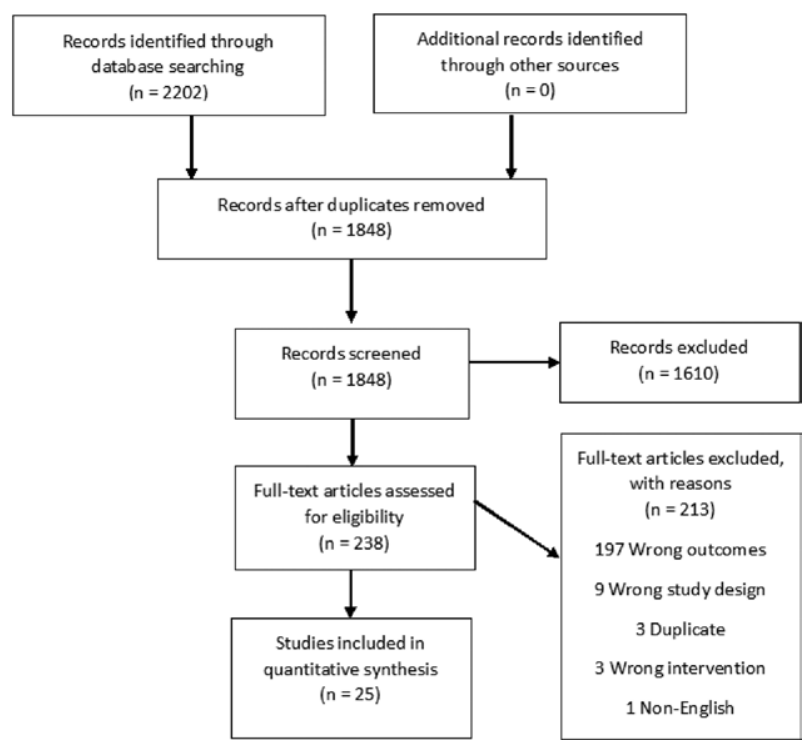

\begin{tabular}{|c|c|c|c|}
\hline & Study & Design & Pso vs HC \\
\hline \multirow{10}{*}{$\begin{array}{l}\text { Bioimpedance } \\
\text { analysis (BIA) }\end{array}$} & Barone et al., 2018 & Cross-sectional & + \\
\hline & Barrea et al., 2015 & Cross-sectional & + \\
\hline & Barrea et al., 2016 & Cross-sectional & + \\
\hline & Demirel et al., 2013 & Cross-sectional & ND \\
\hline & Engin et al., 2014 & Case-control & + \\
\hline & Galluzzo et al, 2017 & Cross-sectional & + \\
\hline & Galluzzo et al, 2018 & Prospective cohort & + \\
\hline & Krajewska-Wlodarczyk et al., 2017 & Cross-sectional & + \\
\hline & Romani, Caixas et al., 2012 & Cross-sectional & + \\
\hline & Romani, Caixas, Cepervelo et al, 2012 & Cross-sectional & + \\
\hline \multirow{6}{*}{$\begin{array}{l}\text { Dual energy X-ray } \\
\text { absorptiometry } \\
\text { (DXA) }\end{array}$} & Diniz Mdos et al., 2016 & Cross-sectional & + \\
\hline & Kofoed et al., 2012 & Cross-sectional & + \\
\hline & Leite et al., 2015 & Cross-sectional & + \\
\hline & Leite et al., 2018 & Randomised control trial & + \\
\hline & Renzo et al., 2011 & Prospective cohort & + \\
\hline & Tournadre et al., 2017 & Cross-sectional & + \\
\hline Ultrasonography & Gonul et al., 2017 & Cross-sectional & + \\
\hline $\begin{array}{l}\text { Transthoracic } \\
\text { echocardiography }\end{array}$ & A kyildiz et al., 2014 & Cross-sectional & + \\
\hline \multirow{2}{*}{$\begin{array}{l}\text { Computed } \\
\text { tomography (CT) }\end{array}$} & Balci et al., 2010 & Cross-sectional & + \\
\hline & Balci et al, 2014 & Cross-sectional & + \\
\hline $\begin{array}{l}\text { Other } \\
\text { Mean muscle index }\end{array}$ & Aguiar et al., 2014 & Cross-sectional & + \\
\hline Body fat (\%) & Andreevskaia et al., 2013 & Cross-sectional & + \\
\hline Body fat (\%) & De La Brassinne et al., 2016 & Cross-sectional & + \\
\hline $\begin{array}{l}\text { Lipid accumulation } \\
\text { product index }\end{array}$ & Ganguly et al., 2016 & Cross-sectional & + \\
\hline $\begin{array}{l}\text { MRI of abdominal fat } \\
\text { distribution }\end{array}$ & Ferguson et al, 2019 & Prospective cohort & + \\
\hline
\end{tabular}

